\title{
New distribution records, first host plant record and DNA barcoding of the Neotropical plume moth Oidaematophorus pseudotrachyphloeus Gielis (Lepidoptera, Pterophoridae)
}

\author{
Héctor A. Vargas ₹ \\ ‡ Universidad Tarapacá, Facultad de Ciencias Agronómicas, Departamento de Recursos Ambientales, Arica, Chile
}

\author{
Corresponding author: Héctor A. Vargas (lepvargas@gmail.com) \\ Academic editor: Shinichi Nakahara \\ Received: 27 Oct 2021 | Accepted: 19 Nov 2021 | Published: 24 Nov 2021 \\ Citation: Vargas HA (2021) New distribution records, first host plant record and DNA barcoding of the \\ Neotropical plume moth Oidaematophorus pseudotrachyphloeus Gielis (Lepidoptera, Pterophoridae). \\ Biodiversity Data Journal 9: e77167. https://doi.org/10.3897/BDJ.9.e77167
}

\begin{abstract}
\section{Background}

Oidaematophorus pseudotrachyphloeus Gielis, 2011 (Lepidoptera, Pterophoridae) is a little-known Neotropical plume moth previously recorded in Ecuador, Peru and Argentina. Its host plants and DNA barcodes are unknown.
\end{abstract}

\section{New information}

Oidaematophorus pseudotrachyphloeus is recorded for the first time from Chile, based on six specimens from the Azapa Valley (Arica Province) and two from Guayacán (Coquimbo Province). Those from the Azapa Valley were reared from folivorous larvae collected on Ambrosia cumanensis Kunth (Asteraceae), representing the first host plant record for this plume moth. The first DNA barcode sequences of $O$. pseudotrachyphloeus are provided and used to explore relationships with congenerics. 


\section{Keywords}

Asteraceae, Atacama Desert, Chile, folivorous larvae, mitochondrial DNA

\section{Introduction}

Oidaematophorus Wallengren, 1862 (Lepidoptera, Pterophoridae, Pterophorinae, Oidaematophorini) is a widespread genus of plume moths with 24 species described (Gielis 2003, Matthews 2005, Gielis 2011a, Gielis 2011b, Hernandez et al. 2014). Species of this genus are recognised by forewing venation with R1 absent, R2, R3, R4 and R5 separate, Cu1 from the posterior angle of the discal cell and $\mathrm{Cu} 2$ from the discal cell, midleg with scale bristles at base of spur pairs and female genitalia with bell- or widened funnel-shaped antrum (Gielis 2011a). Host plants are mainly Asteraceae, but feeding on representatives of Onagraceae and Plantaginaceae has been also documented (Matthews and Lott 2005).

Six species of Oidaematophorus were listed by Gielis (2011a) from the Neotropical Region. Two species were described subsequently, O. espeletiae Hernández, Fuentes, Fajardo \& Matthews, 2014 from Colombia (Hernandez et al. 2014) and O. androsensis Matthews, 2019 from the Bahamas (Matthews et al. 2019), while two others, O. papallacta (Gielis, 2011) and O. praenigratus (Meyrick, 1921), were transferred from Hellinsia Tutt, 1905 by Gielis (2014) and Ustjuzhanin et al. (2021), respectively.

Hitherto, O. pseudotrachyphloeus Gielis, 2011 is reported in Ecuador, Peru and Argentina (Gielis 2011a, Ustjuzhanin et al. 2021). The first records from Chile are reported here. This discovery adds two new localities, provides the first host plant record and allows the sequencing of the first DNA barcodes of this little-known Neotropical plume moth.

\section{Materials and methods}

Plume moths were reared from larvae collected on Ambrosia cumanensis Kunth (Asteraceae) in the Azapa Valley, Arica Province, northern Chile. Eight larvae were collected in July 2017 and placed in plastic vials with leaves of $A$. cumanensis and a paper towel at the bottom. Additional leaves were provided until the larvae completed feeding and pupated, about ten days after collection. Before pupation, two larvae were placed in $95 \%$ ethanol at $-20^{\circ} \mathrm{C}$ until DNA extraction. Adults emerged about twelve days after pupation in August 2017. A search for additional conspecifics from Chile was performed in the Colección Entomológica de la Universidad de Tarapacá (IDEA), Arica, Chile. The abdomen of each specimen was removed and placed in $10 \%$ potassium hydroxide $(\mathrm{KOH})$ for a few minutes for genitalia dissection, stained with Chlorazol black and Eosin and mounted on slides with Euparal. Images of the genitalia were captured with a Sony CyberShot DSCHX200V digital camera attached to a Leica M125 stereomicroscope. The identification of the specimens was based on comparisons with the original description of 0 . pseudotrachyphloeus provided by Gielis (2011a) and photographs of the genitalia slide of 
the male holotype deposited in the Museo de Historia Natural, Universidad Nacional Mayor San Marcos, Lima, Peru (MUSM). The distribution map was generated using SimpleMappr (Shorthouse 2010).

Genomic DNA was extracted from two larvae following the procedures described in Huanca-Mamani et al. (2015). DNA purification, PCR amplification and sequencing of the barcode fragment (Hebert et al. 2003), with the primers LCO-1490 and HCO-2198 (Folmer et al. 1994), were performed in Macrogen Inc. (Seoul, South Korea), following the PCR programme described in Escobar-Suárez et al. (2017). Additional sequences (Table 1) with species identification and 658 base pair length were downloaded from BOLD (Ratnasingham and Hebert 2007) for analysis, including congenerics and representatives of other genera of the tribe Oidaematophorini: Adaina Tutt, 1905, Emmelina Tutt, 1905 and Hellinsia Tutt, 1905. These three genera were used as the outgroup because they are closely related to Oidaematophorus (Gielis 1993). Unfortunately, sequences of Oidaematophorus currently available in public databases are restricted to Nearctic and Palearctic representatives. The sequences were aligned by the ClustalW method and sequence divergence was estimated by the Kimura 2-Parameter (K2P) method in MEGA X (Kumar et al. 2018). Substitution saturation was estimated with the Xia test (Xia et al. 2003) in DAMBE 7 (Xia 2018) to assess the presence of phylogenetic information. A phylogenetic tree was inferred through a Maximum Likelihood (ML) analysis using IQTREE 1.6.12 (Nguyen et al. 2015) in the web interface W-IQ-TREE (Trifinopoulos et al. 2016). Data were partitioned to codon position and $\mathrm{TNe}+\mathrm{I}, \mathrm{F} 81+\mathrm{F}$ and $\mathrm{HKY}+\mathrm{F}+\mathrm{G} 4$ were selected as the best-fit models for 1st, 2 nd and 3rd partitions, respectively, in ModelFinder (Kalyaanamoorthy et al. 2017). Branch support was calculated with 1,000 replications of ultrafast bootstrap (UFBoot, Hoang et al. 2017). The unrooted tree was visualised in FigTree (Rambaut 2014) to root on the representative of Hellinsia, following Gielis (1993).

Table 1.

DNA barcode sequences used in the molecular analysis.

\begin{tabular}{|c|c|c|c|}
\hline Species & $\begin{array}{l}\text { BOLD } \\
\text { accession }\end{array}$ & GenBank accession & Country \\
\hline Oidaematophorus balsamorrhizae McDunnough, 1939 & LNAUS2232-13 & & USA \\
\hline Oidaematophorus brucei (Fernald, 1898) & BBLWU081-09 & $\underline{\mathrm{HM} 428463}$ & USA \\
\hline Oidaematophorus cineraceus (Fish, 1881) & LNAUS2275-13 & & USA \\
\hline Oidaematophorus giganteus (Mann, 1855) & PHLAD170-11 & $\underline{K X 042801}$ & France \\
\hline Oidaematophorus grisescens (Walsingham, 1880) & LNAUS2312-13 & & USA \\
\hline Oidaematophorus guttatus (Walsingham, 1880) & LNAUS2276-13 & & USA \\
\hline Oidaematophorus lindseyi McDunnough, 1923 & LPMNB536-09 & KM550521 & Canada \\
\hline Oidaematophorus lithodactyla (Treitschke, 1833) & LEATE533-13 & & Italy \\
\hline Oidaematophorus mathewianus (Zeller, 1874) & JSJUL1684-11 & KT126373 & Canada \\
\hline Oidaematophorus pseudotrachyphloeus Gielis, 2011 & & $\underline{O K 510535}$ & Chile \\
\hline Oidaematophorus rogenhoferi (Mann, 1871) & LEALT007-16 & MG522712 & Russia \\
\hline
\end{tabular}




\begin{tabular}{|c|c|c|c|}
\hline Species & $\begin{array}{l}\text { BOLD } \\
\text { accession }\end{array}$ & GenBank accession & Country \\
\hline Oidaematophorus vafradactylus Svensson, 1966 & LEFIL230-10 & $\underline{\mathrm{KTT}} 82517$ & Estonia \\
\hline Adaina microdactyla (Hübner, [1813]) & ABOLA573-14 & & Austria \\
\hline Emmelina monodactyla Linneaus, 1758 & FBLMT634-09 & GU706791 & Germany \\
\hline Hellinsia osteodactylus (Zeller, 1841) & ABOLA920-15 & & Austria \\
\hline
\end{tabular}

A
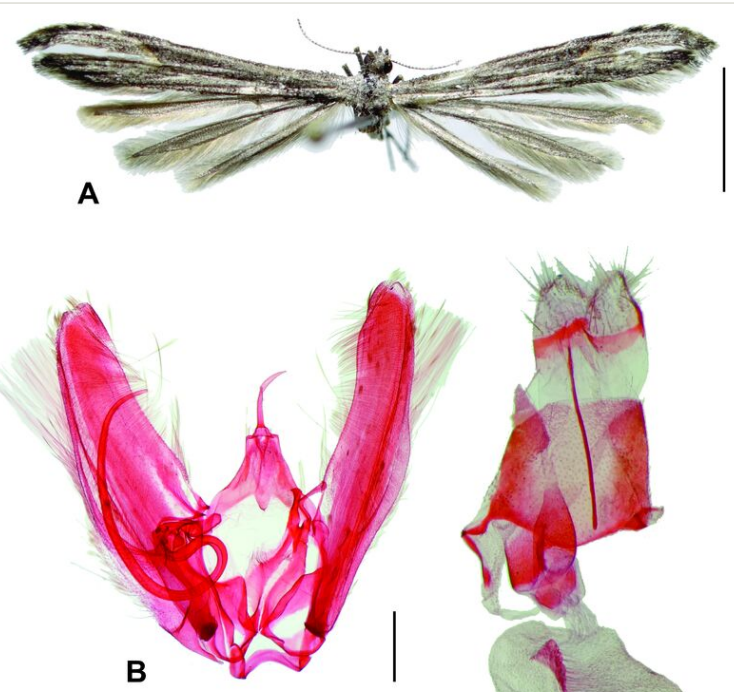

B

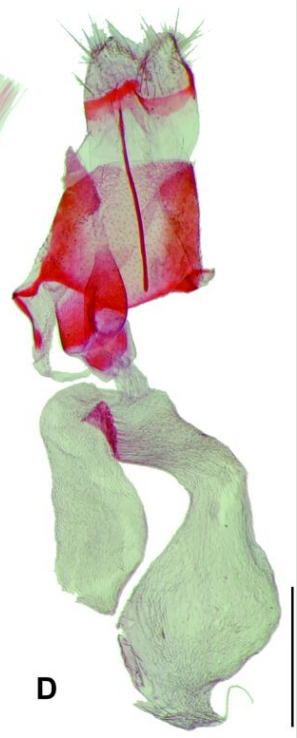

Figure 1. doi

Oidaematophorus pseudotrachyphloeus Gielis, 2011 from Chile. A Female adult in dorsal view; B Male genitalia in ventral view, phallus removed; C Phallus in dorsal view; D Female genitalia in ventral view. Scale bars $5,0.5,0.5,0.5 \mathrm{~mm}$, respectively.

\section{Taxon treatment}

\section{Oidaematophorus pseudotrachyphloeus Gielis, 2011}

\section{Materials}

a. scientificName: Oidaematophorus pseudotrachyphloeus; order: Lepidoptera; family: Pterophoridae; taxonRank: species; scientificNameAuthorship: Gielis, 2011; continent: South America; country: Chile; stateProvince: Arica; locality: Azapa Valley km 12; verbatimCoordinates: $18^{\circ} 31^{\prime} 16^{\prime \prime}$ S 70¹0'42”W; samplingProtocol: One female adult 
emerged August 2017, reared from larva collected on Ambrosia cumanensis July 2017; year: 2017; month: August; sex: female; lifeStage: adult; otherCatalogNumbers: genitalia slide HAV1087; identifiedBy: H.A. Vargas; dateldentified: October 2021; type: PhysicalObject; institutionCode: IDEA; basisOfRecord: PreservedSpecimen

b. $\quad$ scientificName: Oidaematophorus pseudotrachyphloeus; order: Lepidoptera; family: Pterophoridae; taxonRank: species; scientificNameAuthorship: Gielis, 2011; continent: South America; country: Chile; stateProvince: Arica; locality: Azapa Valley km 12; verbatimCoordinates: $18^{\circ} 31^{\prime} 16^{\prime \prime}$ ' $70^{\circ} 10^{\prime} 42^{\prime \prime} \mathrm{W}$; samplingProtocol: One female adult emerged August 2017, reared from larva collected on Ambrosia cumanensis July 2017; year: 2017; month: August; sex: female; lifeStage: adult; otherCatalogNumbers: genitalia slide HAV1090; identifiedBy: H.A. Vargas; dateldentified: October 2021; type: PhysicalObject; institutionCode: IDEA; basisOfRecord: PreservedSpecimen

c. scientificName: Oidaematophorus pseudotrachyphloeus; order: Lepidoptera; family: Pterophoridae; taxonRank: species; scientificNameAuthorship: Gielis, 2011; continent: South America; country: Chile; stateProvince: Arica; locality: Azapa Valley km 12; verbatimCoordinates: $18^{\circ} 31^{\prime} 16^{\prime \prime} \mathrm{S} 70^{\circ} 10^{\prime} 42^{\prime \prime} \mathrm{W}$; samplingProtocol: One female adult emerged August 2017, reared from larva collected on Ambrosia cumanensis July 2017; year: 2017; month: August; sex: female; lifeStage: adult; otherCatalogNumbers: genitalia slide HAV1479; identifiedBy: H.A. Vargas; dateldentified: October 2021; type: PhysicalObject; institutionCode: IDEA; basisOfRecord: PreservedSpecimen

d. scientificName: Oidaematophorus pseudotrachyphloeus; order: Lepidoptera; family: Pterophoridae; taxonRank: species; scientificNameAuthorship: Gielis, 2011; continent: South America; country: Chile; stateProvince: Arica; locality: Azapa Valley km 12; verbatimCoordinates: $18^{\circ} 31^{\prime} 16^{\prime \prime} \mathrm{S} 70^{\circ} 10^{\prime} 42^{\prime \prime} \mathrm{W}$; samplingProtocol: One male adult emerged August 2017, reared from larva collected on Ambrosia cumanensis July 2017; year: 2017; month: August; sex: male; lifeStage: adult; otherCatalogNumbers: genitalia slide HAV1086; identifiedBy: H.A. Vargas; dateldentified: October 2021; type: PhysicalObject; institutionCode: IDEA; basisOfRecord: PreservedSpecimen

e. $\quad$ scientificName: Oidaematophorus pseudotrachyphloeus; order: Lepidoptera; family: Pterophoridae; taxonRank: species; scientificNameAuthorship: Gielis, 2011; continent: South America; country: Chile; stateProvince: Arica; locality: Azapa Valley km 12; verbatimCoordinates: $18^{\circ} 31^{\prime} 16^{\prime \prime} \mathrm{S} 70^{\circ} 10^{\prime} 42^{\prime \prime} \mathrm{W}$; samplingProtocol: One male adult emerged August 2017, reared from larva collected on Ambrosia cumanensis July 2017; year: 2017; month: August; sex: male; lifeStage: adult; otherCatalogNumbers: genitalia slide HAV1089; identifiedBy: H.A. Vargas; dateldentified: October 2021; type: PhysicalObject; institutionCode: IDEA; basisOfRecord: PreservedSpecimen

f. scientificName: Oidaematophorus pseudotrachyphloeus; order: Lepidoptera; family: Pterophoridae; taxonRank: species; scientificNameAuthorship: Gielis, 2011; continent: South America; country: Chile; stateProvince: Arica; locality: Azapa Valley km 12; verbatimCoordinates: $18^{\circ} 31^{\prime} 16^{\prime \prime} \mathrm{S} 70^{\circ} 10^{\prime} 42^{\prime \prime} \mathrm{W}$; samplingProtocol: One male adult emerged August 2017, reared from larva collected on Ambrosia cumanensis July 2017; year: 2017; month: August; sex: male; lifeStage: adult; otherCatalogNumbers: genitalia slide HAV1108; identifiedBy: H.A. Vargas; dateldentified: October 2021; type: PhysicalObject; institutionCode: IDEA; basisOfRecord: PreservedSpecimen

g. scientificName: Oidaematophorus pseudotrachyphloeus; order: Lepidoptera; family: Pterophoridae; taxonRank: species; scientificNameAuthorship: Gielis, 2011; continent: South America; country: Chile; stateProvince: Coquimbo; locality: Guayacán; verbatimCoordinates: $29^{\circ} 57^{\prime} 50^{\prime \prime} S 71^{\circ} 20^{\prime} 51^{\prime \prime} \mathrm{W}$; samplingProtocol: One female adult collected with malaise trap; year: 1976; month: September; sex: female; lifeStage: adult; otherCatalogNumbers: genitalia slide HAV1481; identifiedBy: H.A. Vargas; dateldentified: 
October 2021; type: PhysicalObject; institutionCode: IDEA; basisOfRecord: PreservedSpecimen

h. ScientificName: Oidaematophorus pseudotrachyphloeus; order: Lepidoptera; family: Pterophoridae; taxonRank: species; scientificNameAuthorship: Gielis, 2011; continent: South America; country: Chile; stateProvince: Coquimbo; locality: Guayacán; verbatimCoordinates: $29^{\circ} 57^{\prime} 50^{\prime \prime} \mathrm{S} 71^{\circ} 20^{\prime} 51^{\prime \prime} \mathrm{W}$; samplingProtocol: One male adult collected with malaise trap; year: 1976; month: September; sex: male; lifeStage: adult; otherCatalogNumbers: genitalia slide HAV1480; identifiedBy: H.A. Vargas; dateldentified: October 2021; type: PhysicalObject; institutionCode: IDEA; basisOfRecord: PreservedSpecimen

\section{Taxonomic identification}

Six adults (three females, three males) of O. pseudotrachyphloeus (Fig. 1) were obtained from the larvae collected on A. cumanensis in the Azapa Valley. Two additional conspecifics (one female, one male) from Guayacán (Coquimbo Province) were found in the IDEA collection.

\section{DNA barcoding}

Two identical sequences of O. pseudotrachyphloeus were obtained (GenBank accessions OK510535, OK510536), which represent the first DNA barcodes for this species. The lowest divergence $(9.6 \% \mathrm{~K} 2 \mathrm{P})$ was with O. balsamorrhizae McDunnough, 1939 and O. cineraceus (Fish, 1881). Oidaematophorus was recovered as a monophyletic group in the ML analysis, but with low UFBoot support (Fig. 2). The relationships of $O$. pseudotrachyphloeus with congenerics were not well resolved.

\section{Discussion}

Based on previous records of O. pseudotrachyphloeus (Gielis 2011a, Ustjuzhanin et al. 2021 ), the northern limit of its geographic distribution is found in Loja ( $4^{\circ} 00^{\prime} 30^{\prime \prime} \mathrm{S} ; 79^{\circ} 12^{\prime}$ 42" W; $2030 \mathrm{~m}$ a.s.I.), in the Andes of southern Ecuador and the southern limit is east of the Andes in Córdoba (31 04' 42" S; 64 30' 11" W; 1000 m a.s.I.) , central Argentina,

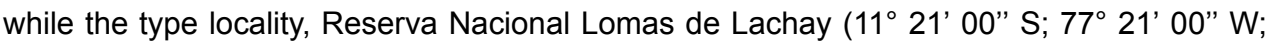
$290 \mathrm{~m}$ a.s.I.), Lima, central Peru, represents the southernmost occurrence west of the Andes. The specimens reported here are the first records of O. pseudotrachyphloeus in Chile. Remarkably, the record from the Azapa Valley (18 $31^{\prime} 16^{\prime \prime} \mathrm{S} ; 70^{\circ} 10^{\prime} 42^{\prime \prime} \mathrm{W} ; 260 \mathrm{~m}$ a.s.I.) reveals that the species is able to breed in the extremely arid Atacama Desert and the record from Guayacán ( $29^{\circ} 57^{\prime} 50^{\prime \prime} \mathrm{S}$; $71^{\circ} 20^{\prime} 51^{\prime \prime} \mathrm{W}$; $30 \mathrm{~m}$ a.s.I.) expands the range of this plume moth by more than $2000 \mathrm{~km}$ to the south along the western margin of South America, exceeding the southern limit of the Atacama Desert (Fig. 3).

Ambrosia cumanensis, erroneously cited as A. peruviana Willd. in the botanical literature (Luebert and Garcia 2020), is the first host plant recorded for O. pseudotrachyphloeus. This discovery agrees with the prevalent association of Oidaematophorus with plants of the family Asteraceae (Matthews and Lott 2005). Ambrosia cumanensis is a widespread 
Neotropical species introduced in Chile, whose only records in this country are restricted to the Azapa Valley (Arica Province) (Moreira-Muñoz et al. 2016). As A. cumanensis is absent in Guayacán (Coquimbo Province), the presence of $O$. pseudotrachyphloeus in this locality suggests that this plume moth would use a different host plant there. The host plant range deserves further attention, as a more detailed knowledge of it could be helpful to understand the geographic distribution of O. pseudotrachyphloeus.

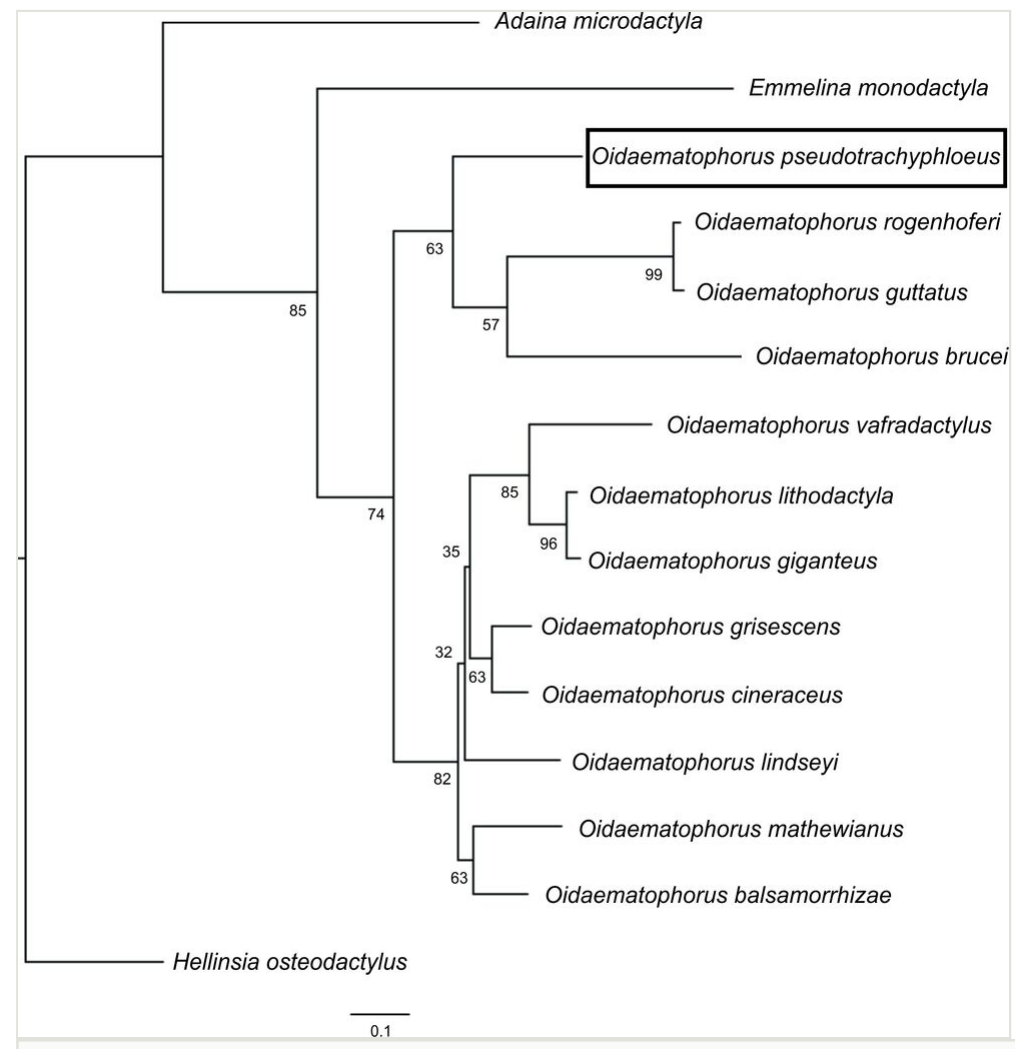

Figure 2. doi

Maximum Likelihood tree of DNA barcodes of Oidaematophorus pseudotrachyphloeus Gielis, 2011 and congenerics. Numbers indicate UFBoot support (\%) of branches.

Although the $\mathrm{ML}$ analysis here presented is based on a single mitochondrial marker, it is promising that sequences of Oidaematophorus were clustered as a monophyletic group in agreement with the most recent morphological definition of the genus (Gielis 2011a). However, the UFBoot support (74\%) of this monophylum was low (Minh et al. 2013). Furthermore, as 0 . pseudotrachyphloeus was the only exclusively Neotropical species included in the alignment, it is not surprising that its relationships were not resolved. A more detailed taxon sampling with emphasis on Neotropical representatives of the genus and the use of additional molecular markers would be needed to understand better the evolutionary relationships of $O$. pseudotrachyphloeus. 
Eight species of Oidaematophorus were previously recorded from Chile (Gielis 1991), all of which were later transferred to Hellinsia, based on morphological evidence (Gielis 2011a): H. betsiae Gielis, 1991, H. cinerarius (Philippi, 1864), H. coquimboicus Gielis, 1991, $H$. grandaevus (Meyrick, 1931), H. hololeucos (Zeller, 1874), H. mallecoicus Gielis, 1991, H. mauleicus Gielis, 1991 and $H$. siskaellus Gielis, 1991. The discovery of $O$. pseudotrachyphloeus confirms the presence of Oidaematophorus in Chile and highlights the importance of surveys for plume moths in the arid environments of the country as suggested by other recent discoveries (Vargas 2020, Vargas et al. 2020, Ustjuzhanin et al. 2021).

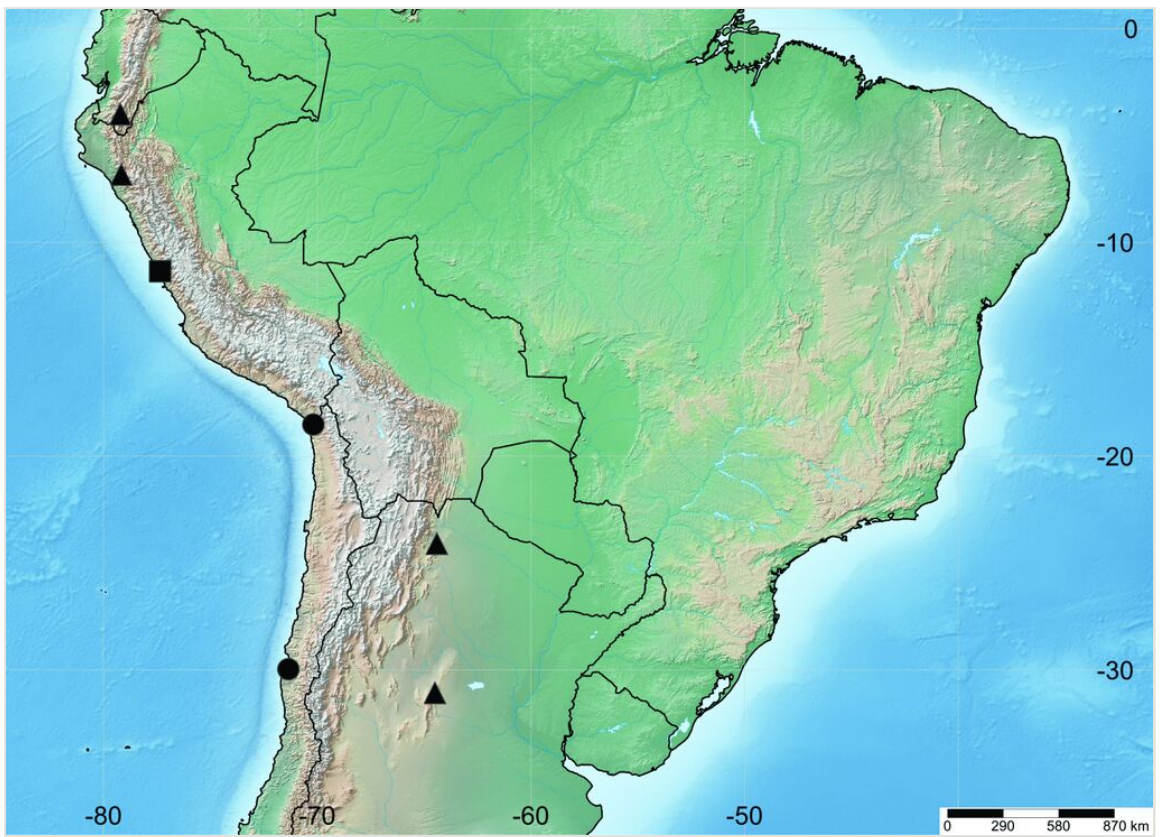

Figure 3. doi

Geographic distribution of Oidaematophorus pseudotrachyphloeus Gielis, 2011 in South America. Square (type locality) and triangles indicate previous records, circles indicate new records from Chile.

\section{Acknowledgements}

The author thanks Maryzender Rodríguez-Melgarejo and Shinichi Nakahara for kind suggestions on a previous version of the manuscript, Bernard Landry and Juan Grados for sharing information and photos of the genitalia slide of the holotype of $O$. pseudotrachyphloeus, respectively, Marcelo Vargas-Ortiz for DNA extraction, Wilson Huanca-Mamani for the use of the molecular biology equipment and Lafayette Eaton for checking the English. 


\section{References}

- Escobar-Suárez S, Huanca-Mamani W, Vargas H (2017) Genetic divergence of a newly documented population of the cecidogenous micromoth Eugnosta azapaensis Vargas \& Moreira (Lepidoptera: Tortricidae) in the Atacama Desert of northern Chile. Revista Brasileira de Entomologia 61 (3): 266-270. https://doi.org/10.1016/j.rbe.2017.05.004

- $\quad$ Folmer O, Black M, Hoeh W, Lutz R, Vrijenhoek R (1994) DNA primers for amplification of mitochondrial cytochrome $\mathrm{c}$ oxidase subunit I from diverse metazoan invertebrates. Molecular Marine Biology and Biotechnology 3 (5): 294-299.

- Gielis C (1991) A taxonomic review of the Pterophoridae (Lepidoptera) from Argentina and Chile. Zoologische Verhandelingen Leiden 269: 1-164.

- $\quad$ Gielis C (1993) Generic revision of the superfamily Pterophoroidea (Lepidoptera). Zoologische Verhandelingen Leiden 290: 1-139.

- $\quad$ Gielis C (2003) World catalogue of insects, 4: Pterophoroidea \& Alucitoidea. Apollo Books, Stenstrup, 198 pp. [ISBN 87-88757-68-4]

- Gielis C (2011a) Review of the Neotropical species of the family Pterophoridae, part II: Pterophorinae (Oidaematophorini, Pterophorini) (Lepidoptera). Zoologische Mededelingen Leiden 85: 1-409.

- $\quad$ Gielis C (2011b) Notes on some African Pterophoridae, with description of new species (Lepidoptera). Boletín de la Sociedad Entomológica Aragonesa 49: 33-63.

- Gielis C (2014) Review of the Neotropical species of the family Pterophoridae, part 5: Additions from Peru, Ecuador, Colombia, Venezuela and the Guyanas (Lepidoptera). Boletín de la Sociedad Entomológica Aragonesa 55: 67-91.

- Hebert PN, Cywinska A, Ball S, deWaard J (2003) Biological identifications through DNA barcodes. Proceedings of the Royal Society of London. Series B: Biological Sciences 270 (1512): 313-321. https://doi.org/10.1098/rspb.2002.2218

- Hernandez LC, Fuentes LS, Fajardo GE, Matthews DL (2014) A new species of Oidaematophorus (Lepidoptera: Pterophoridae) from Chingaza National Natural Park in Colombia. Tropical Lepidoptera Research 24 (1): 15-21.

- Hoang DT, Chernomor O, von Haeseler A, Minh BQ, Vinh LS (2017) UFBoot2: Improving the Ultrafast Bootstrap Approximation. Molecular Biology and Evolution 35 (2): 518-522. https://doi.org/10.1093/molbev/msx281

- Huanca-Mamani W, Rivera-Cabello D, Maita-Maita J (2015) A simple, fast, and inexpensive CTAB-PVP-silica based method for genomic DNA isolation from single, small insect larvae and pupae. Genetics and Molecular Research 14 (3): 8001-8007. https://doi.org/10.4238/2015.july.17.8

- $\quad$ Kalyaanamoorthy S, Minh BQ, Wong TKF, von Haeseler A, Jermiin LS (2017) ModelFinder: fast model selection for accurate phylogenetic estimates. Nature Methods 14 (6): 587-589. https://doi.org/10.1038/nmeth.4285

- Kumar S, Stecher G, Li M, Knyaz C, Tamura K (2018) MEGA X: Molecular evolutionary genetics analysis across computing platforms. Molecular Biology and Evolution 35 (6): 1547-1549. https://doi.org/10.1093/molbev/msy096

- Luebert F, Garcia N (2020) Notas sobre el género Ambrosia (Asteraceae: Ambrosiinae) en Chile. Chloris Chilensis 23 (1): 77-83.

- Matthews D (2005) Pterophoridae of North America. https://www.plumemoth.com/. Accessed on: 2021-11-15. 
- Matthews DL, Lott TA (2005) Larval hostplants of the Pterophoridae (Lepidoptera: Pterophoroidea). Memoirs of the American Entomological Institute 76: 1-324.

- Matthews DL, Miller JY, Simon MJ, Goss GJ (2019) Additions to the plume moth fauna of the Bahamas (Lepidoptera: Pterophoridae) with description of four new species. Insecta Mundi 0708: 1-35.

- $\quad$ Minh BQ, Nguyen MAT, von Haeseler A (2013) Ultrafast Approximation for Phylogenetic Bootstrap. Molecular Biology and Evolution 30 (5): 1188-1195. https://doi.org/10.1093/ molbev/mst024

- Moreira-Muñoz A, Muñoz-Schick M, Marticorena A, Morales V (2016) Catálogo de Asteraceae (Compositae) de la Región de Arica y Parinacota, Chile. Gayana Botánica 73: 226-267. https://doi.org/10.4067/S0717-66432016000200226

- $\quad$ Nguyen L, Schmidt H, von Haeseler A, Minh BQ (2015) IQ-TREE: A Fast and Effective Stochastic Algorithm for Estimating Maximum-Likelihood Phylogenies. Molecular Biology and Evolution 32 (1): 268-274. https://doi.org/10.1093/molbev/msu300

- $\quad$ Rambaut A (2014) FigTree v1.4.2, a graphical viewer of phylogenetic trees. 1.4.2. Institute of Evolotionary Biology, University of Edinburgh, Edinburgh.

URL: http://tree.bio.ed.ac.uk/software/figtree/

- Ratnasingham S, Hebert PD (2007) BOLD: The barcode of life data system (http://www.barcodinglife.org). Molecular Ecology Notes 7 (3): 355-364. https://doi.org/ 10.1111/j.1471-8286.2007.01678.x

- Shorthouse DP (2010) SimpleMappr, an online tool to produce publication-quality point maps. https://www.simplemappr.net. Accessed on: 2021-10-08.

- Trifinopoulos J, Nguyen L, von Haeseler A, Minh BQ (2016) W-IQ-TREE: a fast online phylogenetic tool for maximum likelihood analysis. Nucleic Acids Research 44 https://doi.org/10.1093/nar/gkw256

- Ustjuzhanin PY, Kovtunovich VN, Yakovlev RV, Streltzov AN (2021) Materials on the Lepidoptera fauna of "Ecoportal de Piedra" National Park (Jujuy Province, NorthWestern Argentina). Part I. Pterophoridae (Lepidoptera: Pterophoridae). SHILAP Revista de Lepidopterología 49 (195): 435-446.

- Vargas H (2020) Adaina jobimi sp. nov., a plume moth (Lepidoptera, Pterophoridae) associated with Baccharis alnifolia (Asteraceae) in the Andes of northern Chile. Nota Lepidopterologica 43: 329-336. https://doi.org/10.3897/nl.43.57965

- Vargas H, Vargas-Ortiz M, Gielis C (2020) A new species of Stenoptilia Hübner (Lepidoptera: Pterophoridae) associated with Neobartsia peruviana (Orobanchaceae) in the Andes of northern Chile. Revista Brasileira de Entomologia 64 (2): e20190028.

https://doi.org/10.1590/1806-9665-rbent-2019-0028

- Xia X, Xie Z, Salemi M, Chen L, Wang Y (2003) An index of substitution saturation and its application. Molecular Phylogenetics and Evolution 26 (1): 1-7. https://doi.org/ 10.1016/s1055-7903(02)00326-3

- $\quad$ Xia X (2018) DAMBE7: New and Improved Tools for Data Analysis in Molecular Biology and Evolution. Molecular Biology and Evolution 35 (6): 1550-1552. https://doi.org/ 10.1093/molbev/msy073 